Bull. Austral. Math. Soc.

VOL. 53 (1996) [281-292]

\title{
DIOPHANTINE EQUATIONS OF ERDÖS-MOSER TYPE
}

\author{
Pieter MOREe
}

Using an old result of Von Staudt on sums of consecutive integer powers, we shall show by an elementary method that the Diophantine equation $1^{k}+2^{k}+\cdots+$ $(x-1)^{k}=a x^{k}$ has no solutions $(a, x, k)$ with $k>1, x<10^{10^{6}}$. For $a=1$ this equation reduces to the Erdös-Moser equation and the result to a result of Moser. Our method can also be used to deal with variants of the equation of the title, and two examples will be given. For one of them there are no integer solutions with $x<10^{10^{11}}$.

\section{INTRODUCTION}

The Diophantine equation $1^{k}+2^{k}+\cdots+(x-1)^{k}=x^{k}$ is known as the ErdösMoser equation. An obvious solution is $1+2=3$. Notice that there are no further solutions with $k=1$. Erdös conjectured that there are no solutions with $k>1$. This is Problem D7 in Guy [5], where some historical background is given. It has not been proved that the number of solutions of the Erdös-Moser equation is finite, but if solutions with $k>1$ exist, both $x$ and $k$ must be larger than $10^{10^{6}}$. For $x$ this was proved by Moser [9]. Using Proposition 2 below the same bound can be deduced for $k$. Best and te Riele [1] proved that for every $k$ there is at most one $x$ (the trivial bound is $k+1$ ). The sum $1^{k}+2^{k}+\cdots+(x-1)^{k}$ cries out to be rewritten in terms of Bernoulli polynomials. Urbanowicz [13] seems to have been the first to attack the problem in this way. His approach is continued in [8], where the divisibility properties of solutions with $k>1$ are investigated. It is shown there that $\operatorname{lcm}(1,2 \ldots, 200)$ divides $k$ and that $x$ is not divisible by any regular prime nor by any prime $<10000$.

The present note is more in line with Moser's approach than with the approach of [8] and deals with Erdös-Moser type equations, that is equations of the form $1^{k}+2^{k}+$ $\cdots+(x-1)^{k}=f(x, k)$. Moser's paper is remarkable in that he arrives at such a strong result using only elementary methods. His original paper is a gem of mathemagics. Seemingly out of nothing Moser pulls the four inequalities given in (11) and (12) below

Received 7th June, 1995

This research was carried out at Princeton University. The author thanks The Netherlands Organization for Scientific Research (NWO) for making his stay there possible. Furthermore he wishes to thank A. Granville, J.-L. Nicolas, P. Pleasants, H. te Riele and J. Urbanowicz for their assistance.

Copyright Clearance Centre, Inc. Serial-fee code: 0004-9729/96 \$A2.00+0.00. 
with $a=1$. Adding them together he finds Lemma 1 with $a=1$. This is a strong inequality, for $\sum_{p \mid x} 1 / p$ grows very slowly with $x$. Using a little bit of analytic number theory Moser then deduces that $x \geqslant 10^{10^{\theta}}$. Questions that arise are whether there are more inequalities like those in (11) and (12) (which could give rise to an even larger bound) and whether there is an interconnection between these inequalities. In this note a method will be presented that sheds some light on these questions. Furthermore, the method is sufficiently systematic to allow one to deal with equations of the form

$$
1^{k}+2^{k}+\cdots+(x-1)^{k}=f(x, k) .
$$

We shall study the case $f(x, k)=a x^{k}$ in detail, where we consider $a$ as a variable. Apparently Krzysztofek [6] was the first to consider this equation. Our main result is the following generalisation of Moser's result:

THEOREM 1. The equation

$$
1^{k}+2^{k}+\cdots+(x-1)^{k}=a x^{k}
$$

has no integer solutions $(a, x, k)$ with

$$
k>1, x<\max \left(10^{10^{6}}, a \cdot 10^{22}\right) .
$$

Moser's method can be easily extended to the case of fixed $a$, but it seems harder to generalise to variable $a$. As further examples of our method we shall present in Section 5 results on the equations

$$
1^{k}+2^{k}+\ldots+(x-1)^{k}=a x^{k}+1
$$

and

$$
1^{k}+2^{k}+\cdots+(x-1)^{k}=x^{k}+1+\frac{(x-2)(x-1) x(x+1)(x+5)}{3} 2^{k-6} .
$$

Since our way of dealing with these equations is the same as for (2), we shall indicate only some fine poir.ts of the proofs. Unfortunately for most equations of the form (1), the analogue of Lemma 1 will contain a number on the right hand side of (6) which is too small in order to infer a strong result. Equation (4) is the simplest the author knows of for which a number $>3 \frac{1}{6}$ in the right hand side of $(6)$ can be obtained and thus a larger bound for $x$ than $10^{10^{6}}$ results $\left(10^{10^{11}}\right.$ to be precise).

A key role in our method is played by the identity:

$$
\frac{\sum_{j=1}^{y-1} j^{r}}{y} \equiv\left\{\begin{array}{l}
0\left(\bmod \frac{1}{2}\right) \quad \text { odd } r>1 \\
-\sum_{\substack{p-1|r \\
p| y}} \frac{1}{p}(\bmod 1) \quad r \text { even }
\end{array}\right.
$$


This identity goes back to Von Staudt. We leave it as an exercise for the reader acquainted with Bernoulli numbers to prove (5) by using the Von Staudt-Clausen theorem. A combinatorial proof based on the theory of finite differences was given by Carlitz [3]. A proof of a more general form of (5) using only some results on primitive roots can be found in [7]. For appropriate choices of $y$ in terms of $x$, where $x$ is a solution of $1^{k}+2^{k}+\cdots+(x-1)^{k}=x^{k}$, analysis of (5) yields the four inequalities of Moser.

It is perhaps a tribute to Moser, that the author found (to his surprise) that he could do without Bernoulli numbers. As a demonstration we shall give a more insightful proof of the result that $\operatorname{ord}_{2}(x-3)=\operatorname{ord}_{2}(k)+3$, first established in [8] by using Bernoulli polynomials.

In Section 2 we state and prove the key inequality in the proof of Theorem 1. In Section 3 we show that the fractions in (6) containing $a$ are small and that this implies that $k$ must be large (Lemma 2). In Section 4 we show that the proof of Theorem 1 easily follows from (6) and Lemma 2. In Section 5 we state analogues of Theorem 1 for equations (3) and (4). Finally in Section 6 we prove some further results for the solutions of (2).

\section{The Key LEMMA IN THE PRoOF OF Theorem 1}

By way of numerical computation Theorem 1 will be deduced from the following lemma:

Lemma 1. Suppose $(a, x, k)$ is a solution of (2) with $k>1$. Then

$$
\sum_{\substack{p-1|k \\ p|\left(x^{2}-1\right)\left(4 x^{2}-1\right)}} \frac{1}{p}+\frac{a}{x-1}+\frac{a+1}{x+1}+\frac{2 a}{2 x-1}+\frac{2(a+1)}{2 x+1} \geqslant 3 \frac{1}{6} .
$$

\subsection{The proof of Lemma. 1.}

Proposition 1. Suppose $(a, x, k)$ is a solution of $(2)$ with $k>1$. Then for $b=0$

$$
\frac{a x^{k}}{x+b} \equiv \begin{cases}0\left(\bmod \frac{1}{2}\right) & \text { if } k \text { is odd } \\ -\sum_{\substack{p-1|k \\ p| x}} \frac{1}{p}(\bmod 1) & \text { otherwise. }\end{cases}
$$

For $1 \leqslant b<x$

$$
\frac{a b^{k}-1^{k}-\cdots-(b-1)^{k}}{x-b} \equiv \begin{cases}0\left(\bmod \frac{1}{2}\right) & \text { if } k \text { is odd } \\ -\sum_{\substack{p-1|k \\ p| x-b}} \frac{1}{p}(\bmod 1) & \text { otherwise. }\end{cases}
$$


For $b \geqslant 1$

(7c) $\frac{(a+1)(-b)^{k}+(-1)^{k}\left[1^{k}+\cdots+(b-1)^{k}\right]}{x+b} \equiv \begin{cases}0\left(\bmod \frac{1}{2}\right) & \text { if } k \text { is odd; } \\ -\sum_{\substack{p-1|k \\ p| x+b}} \frac{1}{p}(\bmod 1) & \text { otherwise. }\end{cases}$

Proof: (7a) Put $y=x, r=k$ in (5) and use (2). (7b) $+(7 \mathrm{c})$ For $1 \leqslant b<x$ we have

$$
\begin{aligned}
\sum_{r=0}^{x-b-1} r^{k} & =a x^{k}-\left[(x-1)^{k}+\cdots+(x-b)^{k}\right] \\
& \equiv a b^{k}-\left[1^{k}+2^{k}+\cdots+(b-1)^{k}\right](\bmod x-b)
\end{aligned}
$$

Similarly for $b \geqslant 1$ we have

$$
\begin{aligned}
\sum_{r=0}^{x+b-1} r^{k} & =a x^{k}+x^{k}+(x+1)^{k} \ldots+(x+b-1)^{k} \\
& \equiv(a+1)(-b)^{k}+(-1)^{k}\left[1^{k}+\cdots+(b-1)^{k}\right](\bmod x+b)
\end{aligned}
$$

On taking $y=x-b$ (respectively $x+b(b \geqslant 1))$ in $(5),(7 b)$ (respectively $(7 \mathrm{c})$ ) follows. $\square$

Proposition 1 suggests the importance of determining the parity of $k$. It turns out that $k$ must be even. In the proof we shall use the following bound for $x$ in terms of $a$ and $k$ that is important in its own right:

PROPOSITION 2. If $(a, x, k)$ is a solution of $(2)$, then

$$
a(k+1)<x<(a+1)(k+1) .
$$

ProOF: By comparing the sum $1^{k}+\cdots+(x-1)^{k}$ with $\int t^{k} d t$.

Krzysztofek [6, Lemat 4] has shown by more elaborate but elegant reasoning the stronger result that $a(k+1)+1<x<(a+1 / 2)(k+1)$.

Proposition 3. If $(a, x, k)$ is a solution of $(2)$ with $k>1$, then $k$ is even.

ProOF: Clearly $x \neq 1$, so we may assume $x \geqslant 2$. Suppose $k>1$ and $k$ is odd. Taking $b=1$ in (7b) we see that $2 a /(x-1)$ is integral and in particular that $2 a \geqslant x-1$. But then $2 a \geqslant(k+1) a$, by Proposition 2. This contradiction shows that $k$ must be even.

In the sequel Proposition 3 is used so often that it will not be referred to, but assumed implicitly. 


\section{Proposition 4.}

(i) If the denominator and numerator in (7b) are coprime for some $1 \leqslant b<x$, then $x-b$ is squarefree and $p \mid x-b$ implies $p-1 \mid k$.

(ii) If the denominator and numerator in (7c) are coprime for some $b \geqslant 1$, then $x+b$ is squarefree and $p \mid x+b$ implies $p-1 \mid k$.

Proof: If $p$ divides the denominator of the left hand side, the $p$-order of the fraction is $\leqslant-1$. Since the $p$-order of the right hand side is at least -1 , the $p$-order of both sides is exactly -1 . It follows that $p-1 \mid k$ and that the denominator of the left hand side is squarefree.

EXAMPLES. (i) $(a, b)=(1,1)$ (ii) $(a, b)=\left(2^{m}, x-1\right)(m \geqslant 0),\left(2^{m}-1, x+1\right)(m \geqslant 1)$.

Proposition 5. Suppose $(a, x, k)$ is a solution of (2) with $k>1$. Then

(i) $\left(\frac{1}{2}\right)^{k} \equiv 1\left(\bmod \frac{2 x-1}{\alpha}\right)$ for some divisor $\alpha$ of $a$.

(ii) $\left(\frac{1}{2}\right)^{k} \equiv 1\left(\bmod \frac{2 x+1}{\beta}\right)$ for some divisor $\beta$ of $a+1$.

In (i) and (ii) $1 / 2$ denotes the inverse of 2 modulo $2 x-1$, respectively $2 x+1$.

Proof: Take $b=x-1$ in (7c). On invoking (2) we obtain

$$
\frac{a\left[x^{k}+(x-1)^{k}\right]}{2 x-1} \equiv-\sum_{\substack{p-1|k \\ p| 2 x-1}} \frac{1}{p}(\bmod 1)
$$

Now if $p \mid 2 x-1, p$ is odd and $x \equiv 1 / 2(\bmod 2 x-1)$. The numerator on the left hand side of (8) equals $2 a(1 / 2)^{k}$ modulo $2 x-1$. So we have

$$
\frac{2 a(1 / 2)^{k}}{2 x-1} \equiv-\sum_{\substack{p-1|k \\ p| 2 x-1}} \frac{1}{p}(\bmod 1) .
$$

(i) If $p \mid 2 x-1$ and $p \nmid a$, then from (9) we conclude that $p-1 \mid k$, (so $(1 / 2)^{k} \equiv 1$ $(\bmod p))$ and $p^{2} \nmid 2 x-1$, so we can write

$$
2 x-1=\left(\prod_{\substack{p_{i}\left|\alpha \\ p_{i}\right| 2 x-1}} p_{i}^{\alpha_{i}}\right) \prod_{\substack{p \nmid a \\ p \mid 2 x-1}} p .
$$

Let $a=\prod_{p_{i} \mid a} p_{i}^{\beta_{i}}$ be the prime factorisation of $a$. If $\alpha_{i}>\beta_{i}$, then from (9) it follows that $p_{i}-1 \mid k\left(\right.$ so $\left.(1 / 2)^{k} \equiv 1\left(\bmod p_{i}\right)\right)$ and $\alpha_{i}=\beta_{i}+1$. By the Chinese remainder 
theorem we have

$$
\left(\frac{1}{2}\right)^{k} \equiv 1\left(\bmod \prod_{\substack{p_{i}\left|a \\ \alpha_{i}>\beta_{i} \\ p_{i}\right| 2 x-1}} p_{i} \prod_{\substack{p \nmid a \\ p \mid 2 x-1}} p\right) .
$$

Let $\alpha$ be the quotient of $2 x-1$ by the modulus of the latter congruence. We have

$$
\alpha=\prod_{\substack{p_{i}\left|a \\ \alpha_{i}>\beta_{i} \\ p_{i}\right| 2 x-1}} p_{i}^{\alpha_{i}-1} \prod_{\substack{p_{i}\left|a \\ \alpha_{i} \leqslant \beta_{i} \\ p_{i}\right| 2 x-1}} p_{i}^{\alpha_{i}}
$$

Since $\alpha_{i}=\beta_{i}+1$ whenever $\alpha_{i}>\beta_{i}, \alpha$ is a divisor of $a$.

(ii) Take $b=x+1$ in (7c). Proceeding as in the proof of (i), we obtain

$$
\frac{2(a+1)(1 / 2)^{k}}{2 x+1} \equiv-\sum_{\substack{p-1|k \\ p| 2 x+1}} \frac{1}{p}(\bmod 1)
$$

The rest of the proof is similar to the proof of part (i).

Proof of Lemma 1: Taking $b=1$ in (7b) and (7c) we find that

$$
\sum_{\substack{p-1|k \\ p| x-1}} \frac{1}{p}+\frac{a}{x-1} \geqslant 1, \text { respectively } \sum_{\substack{p-1|k \\ p| x+1}} \frac{1}{p}+\frac{a+1}{x+1} \geqslant 1
$$

On combining (9) and Proposition 5(i), and (10) and Proposition 5(ii), we find that

$$
\sum_{\substack{p-1|k \\ p| 2 x-1}} \frac{1}{p}+\frac{2 a}{2 x-1} \geqslant 1, \text { respectively } \sum_{\substack{p-1|k \\ p| 2 x-1}} \frac{1}{p}+\frac{2(a+1)}{2 x+1} \geqslant 1
$$

Adding the inequalities in (11) and (12) and noting that no prime $>3$ can divide more than one of the numbers $x-1, x+1,2 x-1$ and $2 x+1$ and that 2 and 3 can divide at most two of these numbers, we obtain

$$
\sum_{\substack{p-1|k \\ p|\left(x^{2}-1\right)\left(4 x^{2}-1\right)}} \frac{1}{p}+\frac{a}{x-1}+\frac{a+1}{x+1}+\frac{2 a}{2 x-1}+\frac{2(a+1)}{2 x+1} \geqslant 4-\frac{1}{2}-\frac{1}{3}=3 \frac{1}{6} .
$$




\section{The NONEXISTENCE OF SOLUTIONS With SMALl $k$}

In this section we shall show that (2) has no solutions with $k$ 'small':

Lemma 2. Equation (2) has no solutions with $1<k<10^{22}$.

The number $10^{22}$ is an approximation to twice the 200th highly composite number ( $=2^{8} 3^{5} 5^{3} 7^{2} p_{5} \cdots p_{15}$, where $p_{i}$ denotes the ith successive prime). Recall that Ramanujan defined a number $n$ to be highly composite if $d(n)>d(m)$ for $n>m$, where as usual $d(n)$ denotes the divisor function $\sum_{d \mid n} 1$. A list of the first 5000 highly composite numbers was independently computed by te Riele and Robin. The proof of Lemma 2 will follow on applying Propositions 2 and 6 to (6).

Proposition 6. Let $C>0$ be an arbitrary real number. Suppose $p_{s}$, the sth prime, satisfies

$$
\sum_{p \leqslant p_{s}} \frac{1}{p}<C
$$

Let $n$ be a highly composite number satisfying $d(n) \leqslant s$. Then if $r$ satisfies

$$
\sum_{p-1 \mid 2 r} \frac{1}{p} \geqslant C
$$

the inequality $r \geqslant n$ holds true.

Proof: The number of primes $p$ such that $p-1$ divides $2 r$ is $\leqslant d(r)+1$, so

$$
\sum_{p-1 \mid 2 r} \frac{1}{p} \leqslant \sum_{i=1}^{p_{d(r)+1}} \frac{1}{p_{i}} .
$$

Now if $d(r)<s$ it follows that the sum on the left hand side is $<C$, so $d(r) \geqslant s \geqslant d(n)$. Since $n$ is highly composite it follows that $r \geqslant n$.

Proof of Lemma 2: Using Proposition 2 we see that the sum of the fractions appearing in the left hand side of $(6)$ is $<6 /(k+1)$. Therefore

$$
\sum_{p-1 \mid k} \frac{1}{p}+\frac{6}{k+1}>3 \frac{1}{6} .
$$

For $k=2, \ldots, 998$ it follows by direct computation that this inequality is not satisfied. Since $\sum_{p \leqslant 10^{7}} 1 / p<3.16[9$, Lemma 2$], \pi\left(10^{7}\right)=664579$ and the 200th highly composite number has divisor sum 663552 (the 201th has divisor sum 688128) and exceeds $10^{22}$, the proof of Lemma 2 follows from Proposition 6 with $C=3.16, s=664579$ and $n$ the 200th highly composite number. 


\section{Proof of ThEOREM 1}

Recall that the $a$ dependent terms in (6) are bounded above by $6 /(k+1)$. So on using Lemma 1 and Lemma 2 we find

$$
\sum_{p \mid\left(x^{2}-1\right)\left(4 x^{2}-1\right)} \frac{1}{p}>3.16
$$

Using $\sum_{p \leqslant 10^{7}} 1 / p<3.16$ it follows that $\left(x^{2}-1\right)\left(4 x^{2}-1\right)>\prod_{p \leqslant 10^{7}} p$. Proceeding as in Moser [9] it follows that $x<10^{10^{\theta}}$. For the convenience of the reader we recall his (in essence) argument.

From [12] we have

$$
|\theta(x)-x|<\frac{x}{40 \log x} \quad(x \geqslant 678407)
$$

Hence

$$
\begin{aligned}
\log \left(4 x^{4}\right) & >\log \left(x^{2}-1\right)\left(4 x^{2}-1\right)>\log \prod_{p \leqslant 10^{7}} p=\sum_{p \leqslant 10^{7}} \log p \\
& >\left(1-\frac{1}{280 \log 10}\right) 10^{7}>(.99) 10^{7}, \text { so } x>10^{10^{6}} .
\end{aligned}
$$

On invoking Lemma 2 in combination with Proposition 2, Theorem 1 follows.

\section{Analogues of Theorem 1 for equations (3) and (4)}

Since, in dealing with Erdös-Moser type equations, Proposition 6 has to be applied for various $C$, it is convenient to have estimates for $p_{8}$ and $n$ in this proposition in terms of $C$.

Using the estimate.

$$
\sum_{p \leqslant x} \frac{1}{p}<\log \log x+.2615+\frac{1}{\log ^{2} x} \quad \text { for } x>1
$$

due to Rosser and Schoenfeld $[11,(3.20)]$, we find

$$
\sum_{p \leqslant x} \frac{1}{p}<\log \log x+.267 \text { for } x \geqslant 10^{6} \text {. }
$$

From this inequality one easily deduces: 
Proposition 7. Let $C>2.9$. Inequality (13) is satisfied for any prime $p_{s}$ with

$$
p_{a} \leqslant e^{e^{c-.287}} .
$$

Denote the right hand side of $(17)$ by $f(C)$. Put

$$
g(C)=0.938\{\log f(C)-\log \log f(C)\} \text { and } h(C)=e^{g(C) \log g(C)} .
$$

Using the bound

$$
\frac{\log d(n)}{\log 2} \leqslant 1.537 \frac{\log n}{\log \log n},
$$

of Nicolas and Robin [10], the bound $\pi(x)>x / \log x(x>17)$ of Rosser and Schoenfeld $[11,(3.5)]$ and Proposition 7 we obtain:

Proposition 8. Let $C>2.9$. Suppose $r$ satisfies (14), then $r \geqslant e^{g(C) \log g(C)}$.

Examples.

$$
\begin{aligned}
& f(2.99) \geqslant 4 \cdot 10^{6}, h(2.99) \geqslant 10^{12} . \\
& f(3.16) \geqslant 6 \cdot 10^{7}, h(3.16) \geqslant 10^{16} . \\
& f(3.66) \geqslant 8 \cdot 10^{12}, h(3.66) \geqslant 10^{34} .
\end{aligned}
$$

The proofs of Theorems 2 and 3 are very similar to the proof of Theorem 1 and so we give only sketches. We leave it as an exercise to the reader to complete them.

TheOREM 2. The equation

$$
1^{k}+2^{k}+\cdots+(x-1)^{k}=a x^{k}+1
$$

has no solutions $(a, x, k) \in \mathbb{N}^{3}$ with $k>1, x<\max \left(10^{10^{6}}, a \cdot 10^{12}\right)$.

Proof: (Sketch.) If $x$ is even we have

$$
\sum_{\substack{p-1|k \\ p| x^{3}-x}} \frac{1}{p}+\frac{a+1}{x-1}+\frac{1}{x}+\frac{a+2}{x+1} \geqslant 3
$$

and if $x$ is odd, we have

$$
\sum_{\substack{p-1|k \\ p|\left(x^{2}-2 x\right)\left(x^{2}-1\right)}} \frac{1}{p}+\frac{a}{x-2}+\frac{a+1}{x-1}+\frac{1}{x}+\frac{a+2}{x+1} \geqslant 3 \frac{1}{6} .
$$


After some numerical calculation we conclude that there are no solutions with $1<k<$ $10^{12}<2 h(2.99)$. If $a \leqslant 10^{10^{8}}$, then $x<(a+3 / 2)(k+1)<2^{k}$ and (18) is satisfied irrespective of whether $x$ is even or odd. Now proceed as in the proof of Theorem 1 .

THEOREM 3. The equation

$$
1^{k}+2^{k}+\cdots+(x-1)^{k}=x^{k}+1+\frac{(x-2)(x-1) x(x+1)(x+5)}{3} 2^{k-6}
$$

has no solutions $(x, k) \in \mathbb{N}^{2}$ with $x<10^{10^{11}}$.

Proof: (Sketch.) There are no solutions with $x \leqslant 4$. There are no solutions with $k=1,2$ or 4 . There are no solutions with $k$ even, so we may assume $k \geqslant 6$. Notice that the last term in (19) remains an integer on division by $x+i, i=-2,-1,1$. This remains true if $i=0$ and $3 \nmid x$. We have $k+1<x<(k+1) 3 \frac{1}{4}$. Since $(k+1) 3 \frac{1}{4} \leqslant 2^{k} \leqslant 3^{k}$, it follows from $\sum_{\substack{p-1|k, p| x-3}} 1 / p+3^{k} /(x-3) \equiv 0(\bmod 1)$ that $3 \nmid x$ and from $\sum_{\substack{p-1|k, p| x-2}} 1 / p+2^{k} /(x-2) \equiv 0(\bmod 1)$ that $x$ is odd. We find

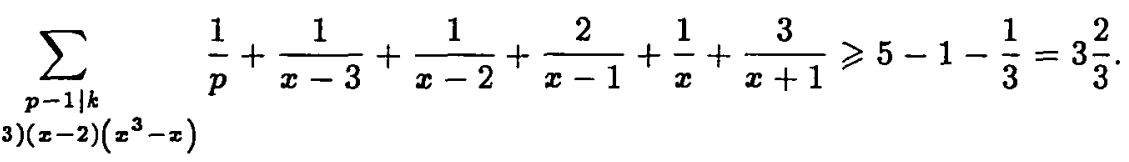

Proceeding as in the proof of Theorem 1 we find

$$
\log \left(x^{5}\right)>\sum_{p \leqslant f(3.66)} \log p>7.99 \cdot 10^{12},
$$

and so $x>10^{10^{11}}$.

Theorems 1, 2 and 3 suggest the following conjecture:

ConjeCture. Equations (2), (3) and (4) do not have integer solutions with $k>1$.

\section{Further Results on (2)}

In this section we derive some further results on the integer solutions of (2) and prove some additional results in the case $a=1$ in (2), that is the Erdös-Moser equation.

Proposition 9. Suppose $(a, x, k)$ with $k>1$ satisfies (2). If $p \mid x$, then $p-1 \nmid k$.

PROOF: From (7a) it follows that $\sum_{\substack{p-1|k, p| x}} 1 / p$ is either zero or a positive integer. Since the latter is clearly impossible, the proposition follows. 
When $a=1$ this result is already known [8, Lemma 10a)].

From Proposition 9 it follows that $x$ must be odd. Using the fact that $k$ is even, the proposition yields that $3 \nmid x$. Take $b=1$ in (7b). Using the fact that $x$ is odd, we see that the 2-order of the right hand side is -1 . So the 2-order of the left hand side must be -1 too, that is, $\operatorname{ord}_{2}(x-1)=\operatorname{ord}_{2}(a)+1$. Suppose $a$ is odd. Then $x \equiv 3$ $(\bmod 4)$. Take $b=3$ in $(7 \mathrm{~b})$. The left hand side is

$$
\frac{a 3^{k}-1-2^{k}}{x-3} \text {. }
$$

Since an easy induction shows that $\operatorname{ord}_{2}\left(3^{r}-1\right)=\operatorname{ord}_{2}(r)+2$ for even $r$ (alternatively one may invoke Proposition 1 of Beyl [2]), we have

$$
a 3^{k}-1-2^{k}=a-1+a m 2^{\text {ord }}(k)+2-2^{k},
$$

where $m$ is odd. Since $k \geqslant 6$, by Lemma 2 , $\operatorname{ord}_{2}(k)+3<k$. We conclude:

Proposition 10. Suppose $(a, x, k)$ satisfies $(2), a$ is odd and $k>1$. Then

$$
\operatorname{ord}_{2}(x-3)= \begin{cases}\operatorname{ord}_{2}(a-1)+1 & \text { if } \operatorname{ord}_{2}(a-1)<\operatorname{ord}_{2}(k)+2 ; \\ \operatorname{ord}_{2}(k)+3 & \text { if } \operatorname{ord}_{2}(a-1)>\operatorname{ord}_{2}(k)+2 ; \\ \geqslant \operatorname{ord}_{2}(k)+4 & \text { otherwise. }\end{cases}
$$

In the case $a=1$ we find $\operatorname{ord}_{2}(x-3)=\operatorname{ord}_{2}(k)+3$, which is Lemma 12 of $[8]$. In the case $a \not \equiv 1(\bmod 8)$, we have ord $2(x-3)=3$ if $a \equiv 5(\bmod 8)$ and 2 otherwise.

For the Erdös-Moser equation the following new result can be proved by comparing orders of the left hand side and right hand side of (7b) with $b=2$ :

Proposition 11. Suppose $(x, k)$ is a solution of the Erdös-Moser equation with $k>1$. Let $p$ be a prime divisor of $x-2$. If 2 is a primitive root modulo $p$, then $p-1 \mid k$ and

$$
\operatorname{ord}_{p}(x-2)=\operatorname{ord}_{p}\left(2^{p-1}-1\right)+\operatorname{ord}_{p}(k)+1 \geqslant 2 .
$$

The following argument works for equations (3) and (4). Take $a$ fixed. By reasoning as in the proof of Proposition 2, we see that there exist positive constants $c_{1}$ and $c_{2}$ such that $c_{1} \leqslant k / x \leqslant c_{2}$. In this case it follows from [4, Théorème 2] that

$$
\sum_{r=1}^{x-1} r^{k}=\frac{(x-1)^{k}}{1-e^{-((k+1) /(x-1))}}\left(1+O\left(\frac{1}{\sqrt{k}}\right)\right) \quad(k \rightarrow \infty) .
$$

From this it is easily deduced that the solutions of equations (3) and (4) satisfy

$$
\lim _{x \rightarrow \infty} \frac{k}{x}=\log \left(1+\frac{1}{a}\right)
$$

that is, when there are infinitely many solutions, the ratio of $k$ and $x$ tends to $\log (1+1 / a)$. When there are only finitely many, since we know that $k$ and $x$ have to be very large the ratio of $k$ to $x$ will be very close to $\log (1+1 / a)$. For $a=1$ this was proved using continued fractions by Best and te Riele [1]. 


\section{REFerences}

[1] M.R. Best and H.J.J. te Riele, On a conjecture of Erdös concerning sums of powers of integers, Technical Report NW 23/76 (Mathematisch Centrum, Amsterdam, 1976).

[2] F.R. Beyl, 'Cyclic subgroups of the prime residue group', Amer. Math. Monthly 84 (1977), 46-48.

[3] L. Carlitz, 'The Staudt-Clausen theorem', Math. Mag. 34 (1961), 131-146.

[4] H. Delange, 'Sur les zéros réels de polynômes de Bernoulli', Ann. Inst. Fourier 41 (1991), 267-309.

[5] R.K. Guy, Unsolved problems in number theory I (Springer-Verlag, Berlin, Heidelberg, New York, 1981).

[6] B. Krzysztofek, 'The equation $1^{n}+\cdots+m^{n}=(m+1)^{n} k^{\prime}$, (in Polish), Wyz. Szkol. Ped. w. Katowicach-Zeszyty Nauk. Sekc. Mat. 5 (1966), 47-54.

[7] P. Moree, 'On a theorem of Carlitz-Von Staudt', C.R. Math. Rep. Acad. Sci. Canada 16 (1994), 166-170.

[8] P. Moree, H.J.J. te Riele and J. Urbanowicz, 'Divisibility properties of integers $x$ and $k$ satisfying $1^{k}+2^{k}+\ldots+(x-1)^{k}=x^{k}$, Math. Comp. 63 (1994), 799-815.

[9] L. Moser, 'On the diophantine equation $1^{n}+2^{n}+\cdots+(m-1)^{n}=m^{n}$, Scripta Math. 19 (1953), 84-88.

[10] J.-L. Nicolas and G. Robin, 'Majorations explicites pour le nombre de diviseurs de $n$ ', Bull. Canad. Math. 26 (1983), 485-492.

[11] J.B. Rosser and L. Schoenfeld, 'A pproximate formulas for some functions of prime numbers', Illinois J. Math. 6 (1962), 64-94.

[12] J.B. Rosser and L. Schoenfeld, 'Sharper bounds for Chebyshev functions $\theta(x)$ and $\psi(x)$ ', Math. Comp. 29 (1975), 243-269.

[13] J. Urbanowicz, 'Remarks on the equation $1^{k}+2^{k}+\cdots+(x-1)^{k}=x^{k}$, Indag. Math. Ser. A 91 (1988), 343-348.

\footnotetext{
Department of Mathematics

Macquarie University

Sydney NSW 2109

Australia
} 